\title{
Ontogenetic changes in the spatial learning capability of jack mackerel Trachurus japonicus.
}

\author{
AUTHOR(S): \\ Takahashi, K; Masuda, R; Yamashita, Y
}

\section{CITATION:}

Takahashi, K ... [et al]. Ontogenetic changes in the spatial learning capability of jack mackerel Trachurus japonicus.. Journal of fish biology 2010, 77(10): 2315-2325

\section{ISSUE DATE:}

2010-11-03

URL:

http://hdl.handle.net/2433/197330

\section{RIGHT:}

This is the peer reviewed version of the following article: Takahashi, K., Masuda, R. and Yamashita, Y. (2010), Ontogenetic changes in the spatial learning capability of jack mackerel Trachurus japonicus. Journal of Fish Biology, 77: 2315-2325, which has been published in final form at [Link to final http://dx.doi.org/10.1111/j.1095-8649.2010.02812.x; この論文は 出版社版でありません。引用の際には出版社版をご確認ご利用ください。; This is not the published version. Please cite only the published version. 
1 Title: Ontogenetic changes in the spatial learning capability of jack mackerel Trachurus

2 japonicus

3

4 Authors: K. Takahashi*, R. Masuda and Y. Yamashita

5 Address: Maizuru Fisheries Research Station, Kyoto University, Nagahama, Maizuru, Kyoto

$6 \quad 625-0086$

$7 \quad *$ Corresponding author

$8 \quad$ Tel: $+81-773-62-5512$

$9 \quad$ Fax: $+81-773-62-5513$

10 Email: tkouji@kais.kyoto-u.ac.jp

11

12 Running head: ONTOGENY OF LEARNING IN JACK MACKEREL

13 


\section{ABSTRACT}

15 Ontogenetic changes in learning capability were studied in jack mackerel Trachurus japonicus (Temminck \& Schlegel) juveniles ranging from 20 to $95 \mathrm{~mm}$ standard length $\left(L_{\mathrm{S}}\right)$

17 collected from either pelagic or coastal habitats. Simple spatial and reversal learning tasks

18 were used to estimate learning capability. There was no size dependence in the scores of

19 simple reward conditioning using a Y-maze, whereas the scores of reversal learning tasks

20 showed a clear sigmoidal curve of increase with an inflexion point at $51.7 \mathrm{~mm} L_{\mathrm{S}}$. The

21 increase of this learning capability coincided with the size at which juveniles recruit from

22 offshore pelagic to coastal rocky habitats.

23 Key words: behavioural ontogeny; cognitive ecology; habitat shift; life history strategy; 
Learning in animals plays an important role in adaptation to changes in environmental

conditions and thus enhances survival potential. Fish have been reported to learn survival

skills in various life history contexts, such as predator avoidance (Brown, 2003; Kelley \&

search and handling (Ehlinger, 1989; Warburton, 2003).

Although the study of learning is well established in freshwater fishes (Coble et al., 1985), relatively few studies have focused on the learning of marine fishes, presumably due to the difficulty of sampling and maintaining them in the laboratory. Previous studies have revealed ontogenetic changes in learning capability through the juvenile stage for some marine fishes such as striped jack Pseudocaranx dentex (Bloch \& Schneider) (Tsukamoto et al., 1995), Pacific threadfin Polydactylus sexfilis (Valenciennes) (Masuda \& Ziemann, 2000) and striped knifejaw Oplegnathus fasciatus (Temminck \& Schlegel) (Makino et al., 2006). Masuda \& Ziemann (2000) proposed that changes in learning capability coincide with ontogenetic shifts in habitat. Indeed most marine fishes experience a major habitat shift and thus face different cognitive challenges during their life history.

All of the above-mentioned studies have examined only hatchery-reared fishes which have never experienced natural environmental conditions. Some of the roles of learning capability (e.g., in relation to predator avoidance, foraging and homing etc.) would better be 
45

evaluated by using wild individuals because hatchery-reared fish may show behavioural inferiority in survival capability such as feeding and anti-predator performance as well as having developmental constraints on learning such as a smaller brain size compared with natural fish (Huntingford, 2004; James et al., 2009). Additionally, the investigation of skills among fish from different life history stages in the natural environment can help us reveal the relationship between learning capability and habitat shift.

Jack mackerel, Trachurus japonicus (Temminck \& Schlegel), one of the most important fisheries resources in Japan, forms large schools and spawns in offshore areas (Sassa et al., 2008). After hatching, larvae drift offshore either independently or associated with floating objects such as seaweed or jellyfish (Sassa et al., 2006). As they reach a standard length of ca. $50 \mathrm{~mm}$, juveniles recruit to rocky coastal reefs (Masuda et al., 2008; Kanaji et al., 2009). In this study, spatial learning capability, including simple spatial learning and its reversal learning tasks, was evaluated in pre and post recruiting stage wild juveniles using a Y-maze. We hypothesized that a shift in learning capability would be associates with recruitment to the spatially complex rocky shore. 


\section{MATERIALS AND METHODS}

Ontogenetic changes in learning capability by reward conditioning were examined in single fish ranging from 20 to $95 \mathrm{~mm} L_{\mathrm{S}}$ (standard length). Fish were captured by three different methods: (1) pelagic fish: collecting individuals associated with drifting objects such as the giant jellyfish Nemopilema nomurai (Kishinouye) (14, 23 Oct and 26 Nov 2007) or drifting algae (18 July 2007) with a hand net while snorkeling in ca. $10 \mathrm{~km}$ offshore area of Maizuru, Kyoto $\left(35^{\circ} 68^{\prime} \mathrm{N}, 135^{\circ} 44^{\prime} \mathrm{E}\right)$, (2) migrating fish: sampled from a set net located ca. $2 \mathrm{~km}$ offshore of Maizuru $\left(35^{\circ} 59^{\prime} \mathrm{N} ; 135^{\circ} 49^{\prime} \mathrm{E}\right.$; 31 July 2007), and (3) coastal fish: captured by artificial fly angling from a pontoon of Maizuru Fisheries Research Station (MFRS) $\left(35^{\circ} 49^{\prime} \mathrm{N}, 135^{\circ} 36^{\prime} \mathrm{E}, 13\right.$ July and 21 Sep 2007). These fish were introduced into separate $500 \mathrm{~L}$ black tanks provided with filtered sea water at an exchange rate of $4 \mathrm{~L}$ per min. They were fed commercial dry pellets (Otohime S2, Syoki-siryou Kyowa N700, Kyowa Hakko Bio Co., Ltd. Tokyo, Japan) and defrosted krill Euphausia sp. from July to December. When fish were confirmed to forage actively on pellets near the water surface, one fish was transferred to an experimental tank and the experiment was started. A total of 40 juveniles $\left(L_{\mathrm{S}} 57 \cdot 7 \pm 20 \cdot 5 \mathrm{~mm}\right.$, mean $\left.\pm \mathrm{SD}\right)$ were used to conduct the experiment: 21 pelagic fish $\left(L_{\mathrm{S}} 42 \cdot 8 \pm 14 \cdot 7 \mathrm{~mm}\right), 11$ migrating fish $\left(L_{\mathrm{S}} 74 \cdot 4 \pm 6 \cdot 0 \mathrm{~mm}\right)$ and 8 coastal fish $\left(L_{\mathrm{S}} 73 \cdot 9 \pm 16 \cdot 4 \mathrm{~mm}\right)$

Five identical glass tanks $(\mathrm{L} \times \mathrm{W} \times \mathrm{H}: 60 \times 30 \times 36 \mathrm{~cm}, 25 \mathrm{~cm}$ in water depth) were 
80

81

prepared as Y-maze experimental tanks for fish $<90 \mathrm{~mm} L_{\mathrm{S}}$. A larger tank $(\mathrm{L} \times \mathrm{W} \times \mathrm{H}: 90 \times$ $30 \times 36 \mathrm{~cm}, 25 \mathrm{~cm}$ in water depth) was used for fish $\geq 90 \mathrm{~mm} L_{\mathrm{S}}(n=3)$, as these individuals did not acclimatize well in the small tank. Each tank was separated into left and right sections by a $20 \times 30(\mathrm{~L} \times \mathrm{H}) \mathrm{cm}(30 \times 30 \mathrm{~cm}$ in the large tank) PVC board set at the center of one of the ends. These two separated zones were designated as the conditioning area (Fig. 1). A removable $\mathrm{PVC}$ board $(\mathrm{W} \times \mathrm{H}: 30 \times 30 \mathrm{~cm})$ was set as the gate at a distance of $20 \mathrm{~cm}$ (30 $\mathrm{cm}$ in the large tank) from the opposite end to the conditioning area, defined as the acclimatization area. Filtered sea water was delivered to the experimental tanks in the conditioning area and drained out from the acclimatization area. Each tank was covered on all sides with a grey sheet to minimize the effects of the observer.

An individual was introduced into the acclimatization area on the day previous to the training and left to acclimatize overnight. A few pellets were provided immediately before a training trial, and if the fish foraged on the pellets, the experiment was started. If not, the experiment was postponed for another day or two. Fish that did not feed after 2 days of acclimatization or did not swim to the conditioning area within 5 min from the time of gate opening were not used in further experiments and regarded as aborting fish. Aborting fish were measured in $L_{\mathrm{S}}$. During the experimental period, fish $\leq 40 \mathrm{~mm} L_{\mathrm{S}}$ were provided with 3-6 small pellets (ca. $0.7 \mathrm{~mm}$ in diameter, Syoki-siryou Kyowa N700) and fish $>40 \mathrm{~mm} L_{\mathrm{S}}$ were provided with 1-5 large pellets (ca. 1.4 mm in diameter, Otohime S2). The amount of 
reward was established by preliminary survey so that fish never satiated during the training.

Feeding was conducted by remote control feeding device, which allowed pellets to drop by pulling a string.

The behaviour of fish was observed after gently opening the gate. Fish were first trained to enter the left side of the branch of a Y maze (original learning task); when the fish swam to the left side, pellets were dropped as the reward into the left side. After feeding on the pellets, the fish was returned gently to the acclimatization area. When the fish swam to the right side, the fish was returned to the acclimatization area without being provided with pellets. The process from opening the gate to returning the fish to the acclimatization area was defined as one trial, and the interval of each trial was about $1 \mathrm{~min}$. Ten trials comprised one session and two consecutive sessions were conducted four times a day at intervals of 30 min or longer. The percentage of choosing the correct side in one session was calculated as the criteria of learning. Once a fish chose the correct side $70 \%$ or more times in three consecutive sessions, it was considered to have learnt the task (Makino et al., 2006). After being conditioned to the original learning task, the fish was conditioned to the reversal learning task where the position of feeding was changed from left to right. When a fish chose the right side $70 \%$ or more times in three consecutive sessions on the reversed task the fish was then again conditioned to an additional reversed task, thus returning the reward side back to the left. Eight sessions a day were carried out for three consecutive days. 
118

119

120

121

122

123

124

125

126

127

128

129

130

131

132

133

134

135

136

Therefore each test fish was conditioned in a total of 24 sessions or 240 trials including the original learning task and all the reversal learning tasks. The reversal learning task was repeated ("right to left" and "left to right") depending on the achievement of the learning task until the end of 240 trainings. The average percentage of the correct choice of side until the accomplishment for each learning task was calculated as a score of each task, and these values were summed to give the total score for each individual. Each fish was anesthetized and measured for $L_{\mathrm{S}}$ after the experiment.

Overall, of all fish indicated a pattern of the total scores increase around $50 \mathrm{~mm} L_{\mathrm{S}}$. Over $50 \mathrm{~mm} L_{\mathrm{S}}$, the scores plateaued and remained constant. Therefore, the relation between $L_{\mathrm{S}}$ and the total score of learning was fitted with a nonlinear least squares model; $y=c+(d-c)$ $/[1+\exp \{-(\mathrm{a}+\mathrm{b} * \mathrm{x})\}]$. Fish were divided and compared as two size groups based upon their differing performance patterns. Total scores were also compared between different habitat groups (i.e. pelagic, migrating or coastal fish) by the Kruskal-Wallis test followed by the Steel-Dwass method.

Personality (bold or shy) of fish may affect learning performance (Sneddon, 2003). Aborting rate was used as a criterion of shyness and was compared in each size and habitat group. Similarly, laterality may also influence learning in spatial tasks (Brown \& Braithwate, 2005; Brown et al., 2004). All the fish were conditioned to the left area on the original learning task, so if there was a difference in laterality with body size, learning score could be 
137 affected by the fish's laterality (Miklosi \& Andrew, 1999; Bisazza et al., 1998). Laterality of

138 individual fish was estimated by the side (left or right) of the first selection on the original

139 learning. Laterality was then compared with the body size. There was a possibility that

140 learning performance was affected by the rearing period because tested fish were kept in

141 captivity for various durations ranging from 5 and 92 days. As plasticity of fish is affected

142 by rearing environment (Berjikian et al., 2001), overly long conventional rearing might have

143 had some effect on the behavioural performance of fish. The effect of rearing period on

144 learning performance was also evaluated by the correlation between rearing period and

145 learning capability.

147 All fish used in experiments were subsequently released into the sea off the MFRS.

148 Small juveniles were kept until they reached $40 \mathrm{~mm} L_{\mathrm{S}}$, because the size range of $T$.

149 japonicus found in this area was between 40 and $120 \mathrm{~mm} L_{\mathrm{S}}$ (Masuda et al., 2008). 


\section{RESULTS}

152

153

154

155

156

157

158

159

160

161

162

163

164

166

167

168

169

The mean $L_{\mathrm{S}}$ of pelagic fish was smaller than those of other groups (Steel-Dwass method, $P<0 \cdot 05$ ). There was no correlation between body size and acclimatization time (Spearman rank method, $r=0 \cdot 09, P=0 \cdot 57)$, nor between the habitats and acclimatization time (Kruskal-Wallis test, $P=0 \cdot 50$ ).

Total learning score followed a logistic curve as follows (Fig. 2): $y=c+(d-c) /[1+$ $\exp \{-(\mathrm{a}+\mathrm{b} * \mathrm{x})\}] ; \mathrm{a}=-47 \cdot 6745(P=0 \cdot 34), \mathrm{b}=0.9222(P=0 \cdot 34), \mathrm{c}=132 \cdot 3460(P<0 \cdot 001)$, $\mathrm{d}=225.2855(P<0 \cdot 001)$. The curve had an inflexion point at $51 \cdot 7 \mathrm{~mm} L_{\mathrm{S}}$ with a score of 178.8. Total score of smaller fish was significantly lower than those of larger size (Mann-Whitney U test, $n=16+24, P<0 \cdot 001$ ). To investigate the relationship between body size and score, the data were divided into two groups with $L_{\mathrm{S}}$ of over and below $51 \cdot 7$ $\mathrm{mm}$. Then there was no correlation between body size and total learning score within each size group (Spearman rank method; $\leq 51 \cdot 7 \mathrm{~mm}: r=0 \cdot 12, P=0 \cdot 65 ;>51 \cdot 7 \mathrm{~mm}: r=0 \cdot 34, P$ $=0 \cdot 10)$. The relation between learning capability and body size was further analysed for each learning task (Fig.3). Reversal learning was repeated for a maximum of four times during the whole trials depending on the achievement of learning criteria. The average score of the original learning task was $80 \cdot 3 \pm 9 \cdot 4$ points, which was achieved on average in $4 \cdot 3 \pm$ 0.8 sessions. There was no size dependence in the score of original learning task $\left(\mathrm{R}_{0}\right)$ and the fourth reversal learning task $\left(\mathrm{R}_{4}\right)\left(\right.$ Spearman rank method, $\mathrm{R}_{0}: r=0 \cdot 24, P=0 \cdot 13, \mathrm{R}_{4}: r=$ 
$0 \cdot 16, P=0 \cdot 32$ ), whereas there was a positive correlation between $L_{\mathrm{S}}$ and score in the first to

171

172

173

174

175

176

177

178

179

180

181

182

183

184

185

186

187

188

third reversal learning tasks $\left(\mathrm{R}_{1}: r=0 \cdot 36, \mathrm{R}_{2}: \mathrm{r}=0 \cdot 65, \mathrm{R}_{3}: \mathrm{r}=0 \cdot 61, P<0 \cdot 05\right)$. The score

showed a clear increase from around $50 \mathrm{~mm}$ in the second and third reversal learning task.

Observation of fish behaviour suggested that smaller fish tended to go directly to the learned area once original learning was established and had difficulty achieveing reversal learning. In contrast, although larger fish also went to the original learning area at the beginning of reversal learning, they tended to stop in front of the conditioning area after several mistakes. Then they started to enter the correct (reversed) side.

The median score of pelagic, migrating and coastal fish were 146·3 (median, IQR (inter quartile range): $123 \cdot 0-180 \cdot 7), 231 \cdot 0(\mathrm{IQR}: 221 \cdot 1-244 \cdot 2)$ and $215 \cdot 5(\mathrm{IQR}: 186 \cdot 0-227 \cdot 3)$, respectively (Fig. 4a). The score of pelagic fish was significantly lower than those of the other two groups (Kruskal-Wallis test followed by Steel-Dwass method, $P<0 \cdot 05$ ). When the scores of fish $>51 \cdot 7 \mathrm{~mm} L_{\mathrm{S}}$ were compared to eliminate the size bias, medians in pelagic $(n=5)$, migrating $(n=11)$ and coastal $(n=8)$ fish were $217 \cdot 3(\mathrm{IQR}: 205 \cdot 4-236 \cdot 9), 231 \cdot 0$ (IQR: $221 \cdot 1-244 \cdot 2$ ) and 215.5 (IQR: $186 \cdot 0$ - 227·3), respectively, and did not differ significantly (Kruskal-Wallis test, $P=0 \cdot 36$; Fig.4b).

A total of 50 fish aborted the experiment: 25 pelagic fish $\left(L_{\mathrm{S}} 42 \cdot 8 \pm 14 \cdot 7 \mathrm{~mm}\right), 16$ migrating fish $\left(L_{\mathrm{S}} 74 \cdot 4 \pm 6 \cdot 0 \mathrm{~mm}\right)$ and 9 coastal fish $\left(L_{\mathrm{S}} 73.9 \pm 16 \cdot 4 \mathrm{~mm}\right)$. There was no significant difference in aborting rate between fish over and below $51.7 \mathrm{~mm}$ (Fisher's exact 
189 test, $P=0 \cdot 83$ ) and among habitats (Fisher's exact test, $P=0 \cdot 92$ ). A total of 25 fish selected

190 the left side and 15 fish selected the right side on the first original learning task. There was

191 no significance difference of the first selection on the original learning task between fish

192 over and below $51.7 \mathrm{~mm}$ (Fisher's exact test, $P=1 \cdot 00$ ). There was no correlation between

193 the rearing duration and learning score in each size group (Spearman rank method, $\leq 51 \cdot 7$

$194 \mathrm{~mm}: r=-0.12 P=0 \cdot 67,>51.7 \mathrm{~mm}: \mathrm{r}=0 \cdot 07, P=0 \cdot 76)$. These findings suggest that these

195 factors do not affect the difference of learning score by size and habitat. 
There was no size-dependence in the original learning score and even the smallest

individuals learned the task. Early juveniles of T. japonicus (size range of ca. 10-50 mm)

often associate with jellyfish and feed on foods collected by jellyfish (Masuda et al. 2008).

The basic spatial learning capabilities represented by the original learning task indicate that

early juveniles may be able to quickly learn the edible parts of jellyfish. Relatively simple

spatial learning skills are likely to be advantageous for survival even in this poor spatial

environment.

reversal learning scores, bigger fish having a higher score than smaller ones within the size second and third reversal learning task, fish above $51 \mathrm{~mm}$ showed a higher score than smaller fish. The total time of reversals indicated a similar tendency, suggesting that reversal that the development of reversal learning capability is related to ontogenetic habitat shift in $T$. rocky reefs area (Masuda et al., 2008; Kanaji et al., 2009). Our study considers reversal

214 learning capability as the ability to quickly learn a novel feeding area in a spatially complex 
216

which lack spatial complexity. Therefore, these results suggest that an increase in reversal learning capability coincides with the recruitment period from the pelagic zone to the rocky reef.

Makino et al. (2006) showed that the learning capability of $O$. fasciatus increases during the transition from offshore to coastal habitat. In this study, $T$. japonicas showed a similar improvement of learning capability during the habitat shift. However, the improvement of learning capability was different in terms of the developmental processes between these species. Learning capability of $O$. fasciatus improved gradually as they grow from 20 to 70 mm Ls. In contrast, learning performance of $T$. japonicas increased suddenly at a threshold size of $51.7 \mathrm{~mm}$. It is tempting to compare the development of brain morphology, which may possibly explain the difference in the improvement trajectories of learning capability in these two species.

In the reversal training, smaller fish tended to continue going directly to the original feeding area. Although larger fish also went to the area of original learning at the beginning of reversal learning, their choice changed after several trials without rewards. These observations suggested that smaller fish learned only the cue, such as a position of feeding, whereas larger fish learned the spatial information of overall feeding area. Small, and thus pelagic, stages of fish may require relatively simple cues for learning feeding areas, whereas cognition of spatial complexity is likely to be necessary for larger coastal fish. Our 
speculation coincides with Odling-Smee et al. (2008) who found that in sympatric species of the stickleback Gasterosteus aculeatus L. species complex, fish from a benthic population in the littoral zone had superior spatial learning abilities to those from a limnetic population in the pelagic zone. The development of learning capability revealed in our study may reflect the behavioural strategy of T. japonicas during its life history as it migrates from pelagic to

240 coastal environments. Hawkins et al. (2008) suggested that in hatchery reared Atlantic salmon Salmo salar L. predator recognition developed at ecologically appropriate periods

242 during ontogeny; three-week-old juveniles showed only an innate response, and 16- to 20-week-old fish showed acquired response to predator odour. Thus, there may be 244 ontogenetic, species specific changes in learning ability in fish. Comparison of learning scores among habitats showed that coastal and migrating fish had a higher learning capability than pelagic fish. This trend supports the hypothesis that the development of learning ability coincides with a habitat shift from offshore pelagic to coastal reefs. However, the result may be biased by the size variation of individuals from each habitat; indeed there was no difference of learning capability among habitats in fish > $51.7 \mathrm{~mm} L_{\mathrm{S}}$. On the other hand, there is a possibility that larger fish sampled from drifting objects were at the last stage of recruitment from pelagic to rocky reef. This was observed during sampling; small juveniles tended to hide inside or behind a floating object, whereas large individuals tended to swim away from the object and headed to the bottom 
254 (Takahashi and Masuda, pers. obs. 2007; Masuda, 2009). It is thus suggested that larger pelagic juveniles are those ready for the transition to rocky reef. The developments of sensory or kinematic organs are often considered as requirements for recruitment (Poling and Fuiman, 1998; Kingsford et al., 2002; Fisher, 2005). The present study suggests that the development of learning capability may also be an important factor for the recruitment to coastal rocky reef. The improvement of learning ability, and thus an increase in adaptability for life in a variable environment, is thus suggested as the factor which enables juveniles to recruit a complex habitat such as coastal rocky shore. history (Yousan, 1988). Present study investigated the ontogeny of learning capability in $T$. japonicus and suggested that these fish were equipped with the appropriate capability in accordance with such changes. Studying the ontogeny of learning capability in fish provides an understanding of the role of learning capability during life history. Further studies should focus on combining environmental factors with learning capability using both wild and hatchery-reared fish. Research on learning capability with various conditioning stimuli may also be required to further investigate learning performance in the context of life history strategies. 
273 juveniles from their set net, Dr. Dominique Robert, Dr. Yuichi Fukunishi and Dr. Michelle L.

274 Walsh helped to improve the manuscript and students of MFRS assisted collection of fish.

275 We also thank Dr. Culum Brown and two reviewers for providing constructive comments on

276 the manuscript.

277 
278

279

280

281

282

283

284

285

286

287

288

289

290

291

292

293

294

295

296

\section{References}

Berejikian, B. A., Tezak, E. P., Riley, S. C. \& LaRae, A. L. (2001). Competitive ability and social behaviour of juvenile steelhead reared in enriched and conventional hatchery tanks and a stream environment. Journal of Fish Biology 59, 1600-1613

Bisazza, A., Rogers, L. J. \& Vallortigara, G. (1998). The origins of cerebral asymmetry: a review of evidence of behavioural and brain lateralization in fishes, reptiles and amphibians. Neuroscience and Biobehavioral Reviews 22, 411-426

Brown, G. E. (2003). Learning about danger: chemical alarm cues and local risk assessment in prey fishes. Fish and Fisheries 4, 227-234

Brown, C. \& Braithwaite, V.A. (2005). Effects of predation pressure on the cognitive ability of the poeciliid Brachyraphis episcope. Behavioural Ecology 16, 482-487

Brown, C., Gardner, C. \& Braithwaite, V.A. (2004). Population variation in lateralized eye use in the poeciliid Brachyraphis episcope. Proceedings of the Royal Society of London Series B 271, S455-S457

Coble, D. W., Farabee, G. B. \& Anderson, R. O. (1985). Comparative learning ability of selected fishes. Canadian Journal of Fisheries and Aquatic Sciences 42, 791-796

Dodson, J. J. (1988). The nature and role of learning in the orientation and migratory behaviour of fishes. Environmental Biology of Fishes 23, 161-182

Ehlinger, T. J. (1989). Learning and individual variation in bluegill foraging: habitat specific 
Fisher, R. (2005). Swimming speeds of larval coral reef fishes: impacts on self-recruitment and dispersal. Marine Ecology Progress Series 285, 223-232

300

301

302

303

304

305

306

307

308

309

310

Hawkins, L. A., Magurran, A. E. \& Armstrong, J. D. (2008). Ontogenetic learning of predator recognition in hatchery-reared Atlantic salmon, Salmo salar. Animal Behaviour 75, 1663-1671

Huntingford, F. A. (2004). Implications of domestication and rearing conditions for the behaviour of cultivated fishes. Journal of Fish Biology 65, 122-142

James, G. B., Arthy, S. \& F. Hellen, R. (2009). Rearing environment affects the brain size of guppies: lab-reared guppies have smaller brains than wild-caught guppies. Ethology $115,122-133$

Kanaji, Y., Watanabe, Y., Kawamura, T., Xie, S., Yamashita, Y., Sassa, C. \& Tsukamoto, Y. (2009). Multiple cohorts of juvenile jack mackerel Trachurus japonicus in waters along the Tsushima Warm Current. Fisheries Research 95, 139-145

Kelley, J. L. \& Magurran, A. E. (2003). Learned predator recognition and antipredator responses in fishes. Fish and Fisheries 4, 216-226

Kingsford, M. J., Leis, J. M., Shanks, A., Lindeman, K. C., Morgan, S. G. \& Pineda, J. (2002). Sensory environments, larval abilities and local self-recruitment. Bulletin of Marine Science 70, 309-340 
Makino, H., Masuda, R. \& Tanaka, M. (2006). Ontogenetic changes of learning capability under reward conditioning in striped knifejaw Oplegnathus fasciatus juveniles. Fisheries Science 72, 1177-1182

Masuda, R. (2009). Ontogenetic changes in the ecological function of the association behavior between jack mackerel Trachurus japonicas and jellyfish. Hydrobiologia 616, 269-277

Masuda, R. \& Ziemann, D. A. (2000). Ontogenetic changes of learning capability and stress recovery in Pacific threadfin juveniles. Journal of Fish Biology 56, 1239-1247

Masuda, R., Yamashita, Y. \& Matsuyama, M. (2008). Jack mackerel Trachurus japonicus juveniles use jellyfish for predator avoidance and as a prey collector. Fisheries Science 74, 276-284

Miklosi, A. \& Andrew, R. J. (1999). Right eye use associated with decision to bite in zebrafish. Behavioural Brain Research 105, 199-205

Odling-Smee, L. \& Braithwaite, A. V. (2003). The role of learning in fish orientation. Fish and Fisheries 4, 235-246

Odling-Smee, L., Boughman, J. W. \& Braithwaite, A. V. (2008). Sympatric species of threespine stickleback differ in their performance in a spatial learning task. Behavioral, Ecology and Sociobiology 62, 1935-1945

Poling, K. R. \& Fuiman, L. A. (1998). Sensory development and its relation to habitat 
Sassa, C. Konishi, Y \& Mori, K. (2006). Distribution of jack mackerel (Trachurus japonicus) larvae and juveniles in the East China Sea, with special reference to the larval transport by the Kuroshio Current. Fisheries Oceanography 15, 508-518

Sassa, C., Tsukamoto, Y., Nishiuchi, K. \& Konishi, Y. (2008). Spawning ground and larval transport processes of jack mackerel Trachurus japonicus in the shelf-break region of the southern East China Sea. Continental Shelf Research 28, 2574-2583

Sneddon, L. U. (2003). The bold and the shy: individual differences in rainbow trout. Journal of fish biology 62, 971-975 change in abilities of learning and memory in striped jack Pseudocaranx dentex. Saibai Giken 24, 45-51

Youson, J. H. (1988). First metamorphosis. In Fish Physiology, Vol. 11B (Hoar, W. S. \& Randall, D. J., eds), pp. 135-196. New York: Academic Press. 


\section{Figure Caption}

Fig.1. Schematic drawing of the experimental tank. A fish was kept in the acclimatization area until the gate was opened. In the original learning task, the fish was rewarded by pellets when it went into the left side. In the reversal learning task, the correct side was reversed from left to right.

Fig.2. Total score including original and all reversal learnings in 240 trials for fish captured from the drifting objects (०), set net $(\bullet)$ and angling $(\square)$. Each marker represents one fish. Solid line represents nonlinear least square model fitted to the data. Model equation is $\mathrm{y}=929395 /\{1+\mathrm{e}(-56 \cdot 37-$ $1 \cdot 15 \mathrm{x})\}+126 \cdot 85$. Dotted line represents the inflexion point of the model.

Fig.3. Score of original and each reversal learning task. (a) original learning, (b) first, (c) second, (d) third and (e) fourth reversal learning. Each line indicates a linear regression line. Individuals that could not accomplish a learning stage were treated as zero score, and black plots indicate such individuals.

Fig. 4. (a) The median of the total score of all fish captured under each sampling method. Bars indicate inter quartile range (drifting objects: $n=21$, set net: $n=11$, angling: $n=8$ ). Scores with different letters were significantly different (Steel-Dwass method, $P<0 \cdot 05$ ). (b) The median of the total score of fish $>51.7 \mathrm{~mm} L_{\mathrm{S}}$ captured under each sampling method. Bars indicate interquartile range (drifting objects: $n=5$, set net: $n=11$, angling: $n=8$ ). 
Figure

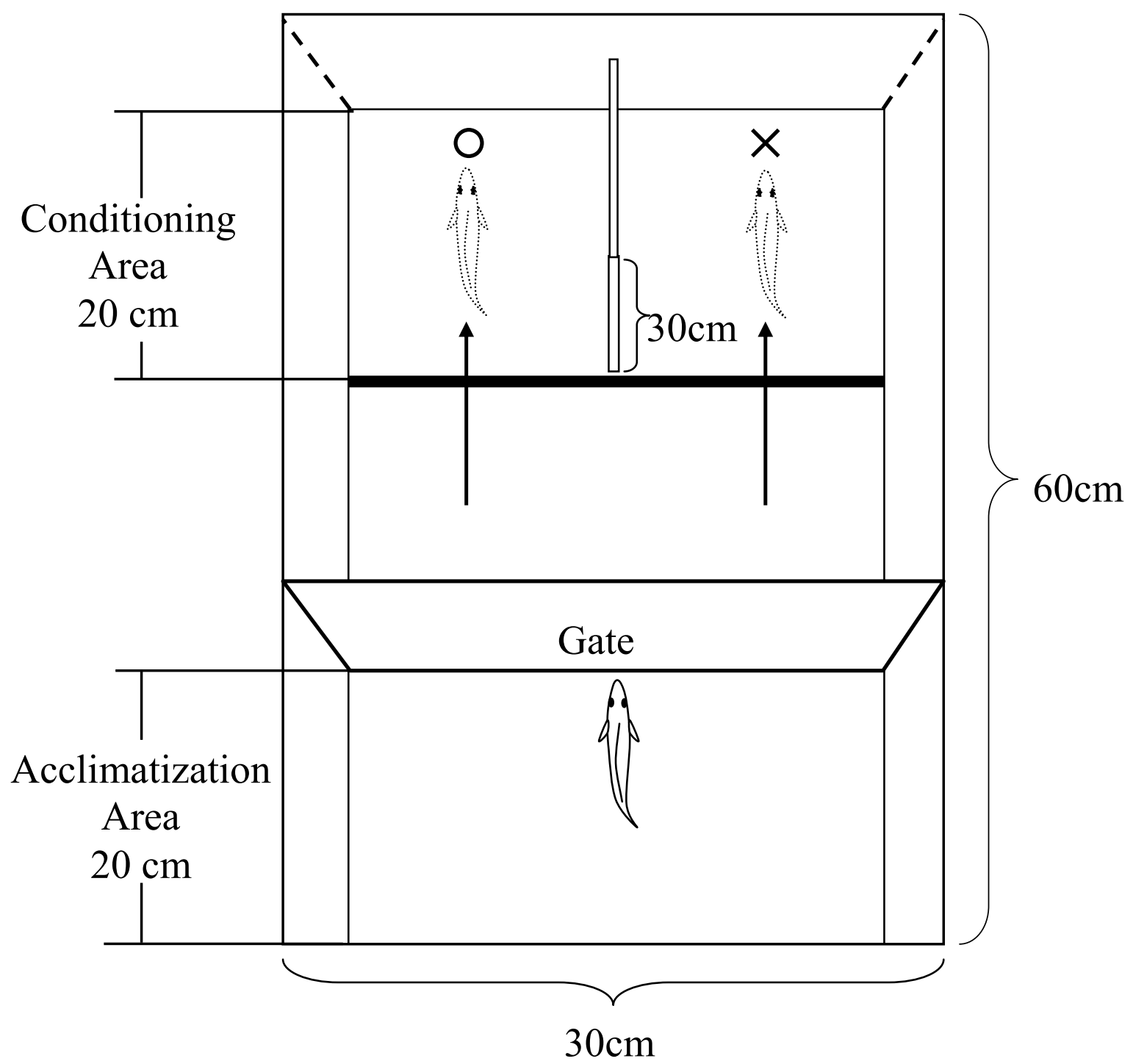

Fig.1 


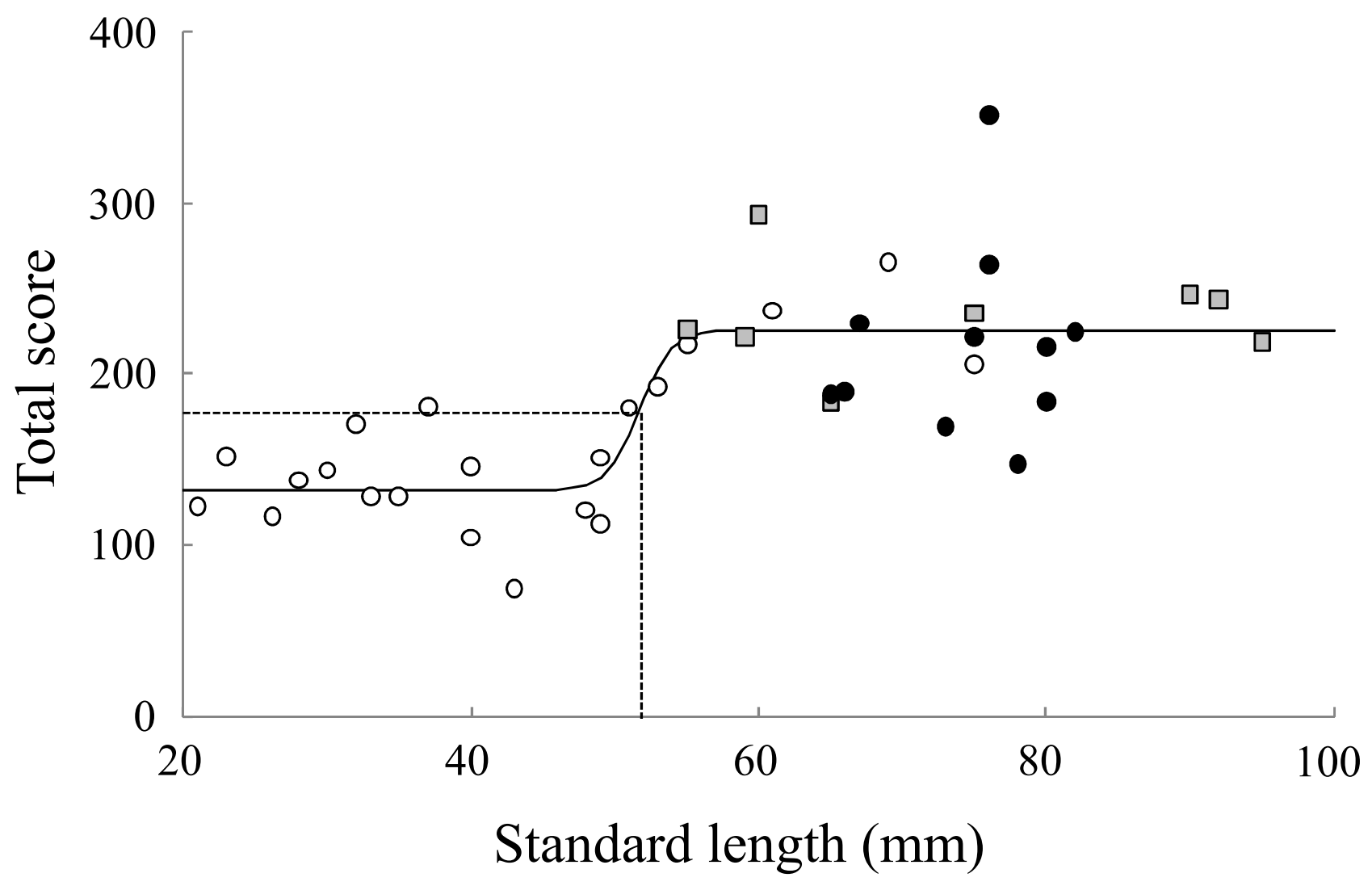

Fig.2 


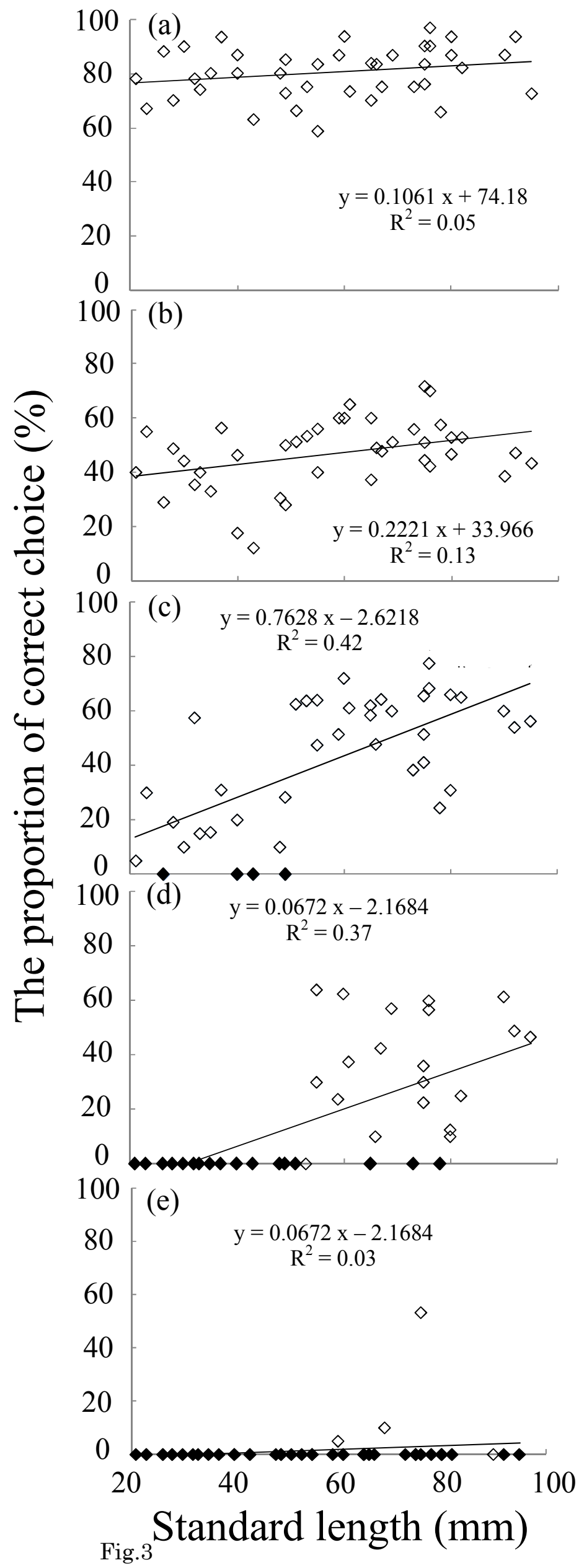




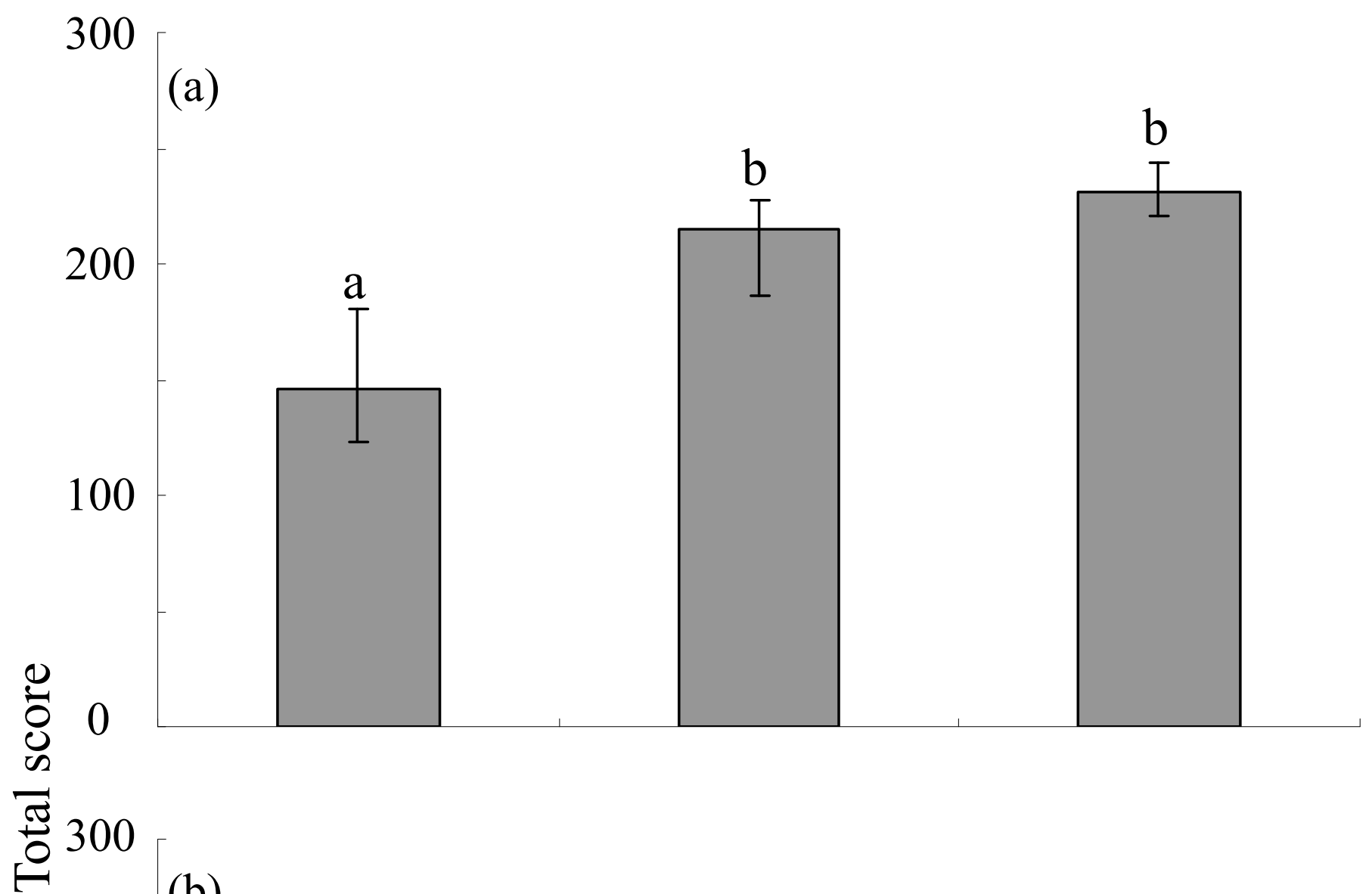

(b)

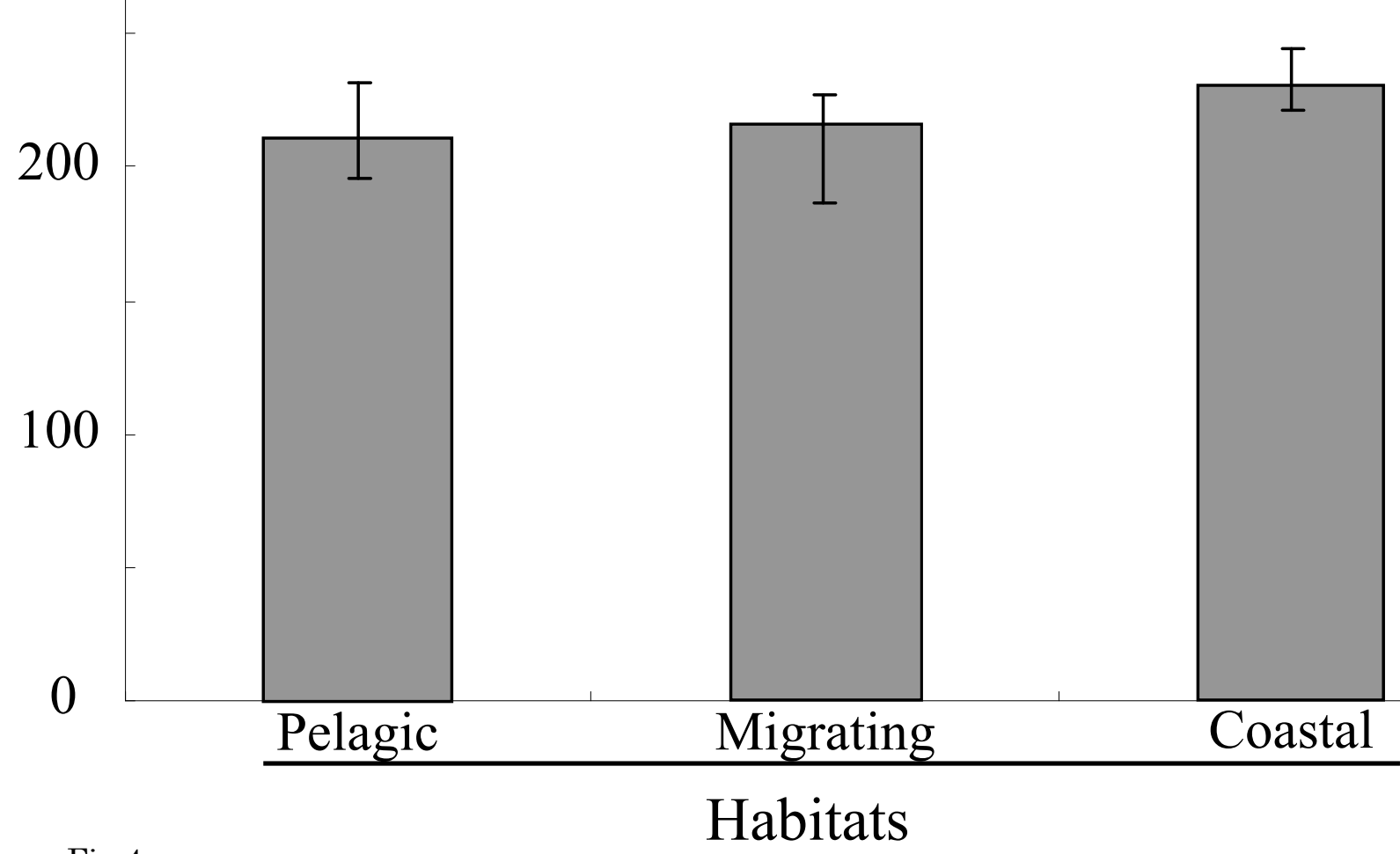

Fig.4 